\title{
Some results in fixed point theory and application to the convergence of some iterative processes
}

\author{
Najeh Redjel ${ }^{1,2^{*}}$ and Abdelkader Dehici, ${ }^{1,2}$
}

\section{"Correspondence:}

najehredjel@yahoo.fr

'Laboratory of Informatics and

Mathematics, University of

Souk-Ahras, P.O. Box 1553,

Souk-Ahras, 41000, Algeria

${ }^{2}$ Department of Mathematics,

University of Constantine 1,

Constantine, 25000, Algeria

\section{然 Springer}

\begin{abstract}
In this paper, we study the existence and uniqueness of fixed points for a class of self-mappings satisfying certain rational expressions on closed, bounded and convex subsets with normal structures in reflexive Banach spaces. We show that, in particular, this class extends that introduced by Ray and Singh (Indian J. Pure Appl. Math. 9:216-221, 1978). As an application, we give an investigation of the convergence and stability of some iterative processes associated to these mappings.
\end{abstract}

MSC: $47 \mathrm{H} 09 ; 47 \mathrm{H} 10 ; 54 \mathrm{H} 25$

Keywords: Banach space; normal structure; rational expression; fixed point; Picard iteration; Mann iteration; Ishikawa iteration; Kirk iteration

\section{Introduction}

It is well known that the Banach contraction principle was the starting point for the development of fixed point theory allowing the advancement of the analysis and nonlinear analysis in particular. During more than fifty years, there has been a lot of production in this area and many well-known fixed theorems have been established and investigated by several authors (see, for example, [1-7] and the references therein).

Definition 1.1 A function $\Phi:[0,+\infty[\longrightarrow[0,+\infty$ [ is called a comparison function if it satisfies the following conditions:

(i) $\Phi$ is monotone nondecreasing;

(ii) $\lim _{n \rightarrow+\infty} \Phi^{n}(t)=0$ for all $t>0$ ( $\Phi^{n}$ stands for the $n$th iterate of $\left.\Phi\right)$.

Remark 1.2 Every comparison function satisfies $\Phi(0)=0$ and $\Phi(t)<t, \forall t>0$.

Fixed point theory has been and will remain an important tool in the study of the existence and uniqueness of solutions of differential and integral equations. Among the extensions of Banach's principle, we quote the fixed point result of Boyd and Wong [8].

Theorem 1.3 [8] Let $(X, d)$ be a complete metric space and $T$ be a self-mapping on $X$ satisfying that

(c) 2015 Redjel and Dehici. This article is distributed under the terms of the Creative Commons Attribution 4.0 International License (http://creativecommons.org/licenses/by/4.0/), which permits unrestricted use, distribution, and reproduction in any medium, provided you give appropriate credit to the original author(s) and the source, provide a link to the Creative Commons license, and indicate if changes were made. 


$$
d(T(x), T(y)) \leq \Phi(d(x, y)) \quad \text { (where } \Phi \text { is a comparison function }) .
$$

Then T has a unique fixed point in $X$.

Other rational expressions generalizing the same principle have been investigated by several authors. Among the more classical ones, those that have been established by Jaggi, Dass and Gupta.

Theorem 1.4 [9] Let $(X, d)$ be a complete metric space and $T: X \longrightarrow X$ be a continuous mapping such that

$$
d(T(x), T(y)) \leq \alpha \frac{d(x, T(x)) d(y, T(y))}{d(x, y)}+\beta d(x, y)
$$

for all distinct points $x, y \in X$, where $\alpha, \beta \in[0,1[$ with $\alpha+\beta<1$. Then $T$ has a unique fixed point.

Theorem 1.5 [10] Let $(X, d)$ be a complete metric space and let $T$ be a mapping from $X$ into itself satisfying

$$
d(T(x), T(y)) \leq \alpha d(y, T(y)) \frac{1+d(x, T(x))}{1+d(x, y)}+\beta d(x, y)
$$

for all distinct points $x, y \in X$, where $\alpha, \beta \in[0,1[$ with $\alpha+\beta<1$. Then Thas a unique fixed point $\xi \in X$. Moreover, for all $x \in X$, the sequence $\left\{T^{n}(x)\right\}$ converges to $\xi$.

Definition 1.6 Let $(X,\|\cdot\|)$ be a normed space and $K$ be a nonempty subset of $X$. A selfmapping $T$ on $K$ is called a nonexpansive mapping if $\|T(x)-T(y)\| \leq\|x-y\|$ for all $x, y \in K$.

The set of nonexpansive self-mappings on $K$ will be denoted by $\Delta_{K}$.

We note that the existence and uniqueness of fixed points for this type of mappings are not in general ensured. However, some fixed point theorems for nonexpansive mappings have been derived by several authors (we quote, for example, [11, 12] and Chapter 3 of [13]).

Let $(X,\|\cdot\|)$ be a normed space and $T$ be a self-mapping on $X$. For any nonempty bounded subset $A \subset X$, the diameter of $A$, which will be denoted by $\delta(A)$, is defined by

$$
\delta(A)=\sup \{\|x-y\|: x, y \in A\} .
$$

Let $x \in X$ and $n \in \mathbb{N}$, we define the orbit of order $n$ of $x$ by $T$ as follows:

$$
O(x, n)=\left\{x, T(x), \ldots, T^{n}(x)\right\} .
$$

The orbit of $x$ by $T$ is defined by

$$
O(x)=\left\{x, T(x), \ldots, T^{n}(x), \ldots\right\}
$$


Definition 1.7 Let $(X,\|\cdot\|)$ be a normed space and $K$ be a nonempty bounded subset of $X$. Let $T: K \longrightarrow K$ be a mapping, $T$ is said to be orbitally controlled if $\forall n \in \mathbb{N}, n \geq 2, \forall x \in X$ and for all $i, j \in\{1, \ldots, n\}$, there exists $1 \leq k \leq n$ such that

$$
\left\|T^{i}(x)-T^{j}(x)\right\| \leq\left\|x-T^{k}(x)\right\| .
$$

Remark 1.8 The integer $k$ indicated in the above definition can be chosen independently of $i, j$. Indeed, let $n \geq 2$ and $x \in K$, and assume that for all $i, j \in\{1, \ldots, n\}$, there exists an integer $k$ (depending on $i, j$ ) such that

$$
\left\|T^{i}(x)-T^{j}(x)\right\| \leq\left\|x-T^{k_{i, j}}(x)\right\| .
$$

If we define $l=\max \left\{\left\|x-T^{k_{i, j}}(x)\right\|, i, j=1, \ldots, n\right\}$, then there exist $i_{0}, j_{0} \in\{1, \ldots, n\}$ such that $l=\left\|x-T^{k_{i_{0}, j_{0}}}(x)\right\|$; consequently, we get

$$
\left\|T^{i}(x)-T^{j}(x)\right\| \leq\left\|x-T^{k_{i_{0}, j_{0}}}(x)\right\|
$$

for all $i, j \in\{1, \ldots, n\}$ (here $k_{i_{0}, j_{0}}$ is independent of $i$ and $j$ ).

If $T$ is orbitally controlled, then for each $x \in K$ and $n \geq 2$, there exist $1 \leq \beta_{1}^{x} \leq n$ and $2 \leq \beta_{2}^{x} \leq n$ such that $\delta(O(x, n))=\left\|x-T^{\beta_{1}^{x}}(x)\right\|$ and $\delta((O(T(x), n-1)))=\left\|T(x)-T^{\beta_{2}^{x}}(x)\right\|$. We denote by $r_{1}^{x}(T)$ (resp. $\left.r_{2}^{x}(T)\right)$ the smallest integer $\beta_{1}^{x}$ (resp. $\left.\beta_{2}^{x}\right)$ such that $\delta(O(x, n))=$ $\left\|x-T^{\beta_{1}^{x}}(x)\right\|\left(\right.$ resp. $\delta(O(T(x), n-1))=\left\|T(x)-T^{\beta_{2}^{x}}(x)\right\|$. We note that $\delta(O(x, n)) \geq \delta(O(T(x)$, $n-1))$.

The set of orbitally controlled self-mappings on $K$ will be denoted by $\Xi_{K}$.

\section{Main results}

First of all, we will show that $\Xi_{K}$ contains in particular the set of all nonexpansive selfmappings $\Delta_{K}$.

Proposition 2.1 Let $(X,\|\cdot\|)$ be a normed space and $K$ be a nonempty bounded subset of $X$. Then $\Delta_{K} \subseteq \Xi_{K}$.

Proof Let $T \in \Delta_{K}$ and let $n \in \mathbb{N}, n \geq 2$. If $x \in K$ and $i, j \in\{1, \ldots, n\}, j>i$, we have

$$
\left\|T^{j}(x)-T^{i}(x)\right\| \leq\left\|T^{j-1}(x)-T^{i-1}(x)\right\| \leq \cdots \leq\left\|x-T^{j-i}(x)\right\|,
$$

which implies that $T$ satisfies (1) (since $1 \leq j-i<n$ ), thus $T \in \Xi_{K}$, which gives the result.

Let us give now the definition of quasi-contraction self-mappings.

Definition 2.2 Let $(X,\|\cdot\|)$ be a normed space and $K$ be a nonempty subset of $X$. A selfmapping $T$ on $K$ is said to be a quasi-contraction mapping if there exists $\eta \in[0,1[$ such that

$$
\|T(x)-T(y)\| \leq \eta \max \{\|x-y\|,\|x-T(x)\|,\|y-T(y)\|,\|x-T(y)\|,\|y-T(x)\|\}
$$

for all $x, y \in K$. 
The set of quasi-contraction self-mappings on $K$ will be denoted by $\Theta_{K}$.

Proposition 2.3 Let $(X,\|\cdot\|)$ be a normed space and $K$ be a nonempty bounded subset of $X$. Then $\Theta_{K} \subseteq \Xi_{K}$.

Proof If $T \in \Theta_{K}$ and $n \geq 2$. Let $x \in K$ and let $1 \leq i, j \leq n$. Thus $T^{i}(x), T^{j}(x) \in O(T(x)$, $n-1) \subset O(x, n)\left(T^{0}(x)=x\right)$. Since $T$ is a quasi-contraction self-mapping on $K$, it follows that

$$
\begin{aligned}
\left\|T^{i}(x)-T^{j}(x)\right\|= & \left\|T\left(T^{i-1}(x)\right)-T\left(T^{j-1}(x)\right)\right\| \\
\leq & \eta \max \left\{\left\|T^{i-1}(x)-T^{j-1}(x)\right\|,\left\|T^{i-1}(x)-T^{i}(x)\right\|,\left\|T^{j-1}(x)-T^{j}(x)\right\|,\right. \\
& \left.\left\|T^{i-1}(x)-T^{j}(x)\right\|,\left\|T^{j-1}(x)-T^{i}(x)\right\|\right\} \\
\leq & \eta \delta(O(x, n))<\delta(O(x, n)) .
\end{aligned}
$$

This shows that (1) is satisfied for $T$ and, consequently, $\Theta_{K} \subseteq \Xi_{K}$.

Lemma 2.4 Let $(X,\|\cdot\|)$ be a normed space and $K$ be a nonempty subset of $X$. Let $n \in \mathbb{N}$, $n \geq 2$, if $T \in \Delta_{K}$ and $x \in K$ such that $\delta(O(x, n))=\delta(O(T(x), n-1))$, then $r_{2}^{x}(T)>r_{1}^{x}(T)$.

Proof Assume that $r_{2}^{x}(T) \leq r_{1}^{x}(T)$. By hypothesis, we obtain that $\delta(O(x, n))=\delta(O(T(x)$, $n-1))=\left\|T(x)-T_{2}^{r_{2}^{x}(T)}(x)\right\|=\left\|x-T_{1}^{r_{1}^{x}(T)}(x)\right\|$. The fact that $T \in \Delta_{K}$ implies that $\delta(O(x, n))=$ $\delta(O(T(x), n-1))=\left\|T(x)-T_{2}^{r_{2}^{x}(T)}(x)\right\| \leq\left\|x-T_{2}^{r_{2}^{x}(T)-1}(x)\right\|$, which is a contradiction with $r_{1}^{x}(T)$ is the smallest integer $\beta_{1}^{x}$ such that $\delta(O(x, n))=\left\|x-T^{\beta_{1}^{x}}(x)\right\|$.

Let $P$ be a real polynomial given by

$$
P(x)=\lambda_{0}+\lambda_{1} x+\cdots+\lambda_{n} x^{n} \quad \text { with } \lambda_{i} \geq 0, \lambda_{1}>0 \text {, and } \sum_{i=0}^{n} \lambda_{i}=1 .
$$

Let $(X,\|\cdot\|)$ be a normed space and $K$ be a nonempty subset of $X$. Let $T: K \longrightarrow K$ be a self-mapping. By $F(T)$, we will designate the set of fixed points of $T$ in $K$, the convex hull of $K$ is denoted by $\operatorname{co}(K)$ and the closed convex hull of $K$ is denoted by $\overline{\operatorname{co}}(K)$.

Proposition 2.5 If $K$ is a nonempty, bounded and convex subset in $X$ and $T \in \Xi_{K}$ such that $r_{2}^{x}(T)>r_{1}^{x}(T)$ for all $x \in K$, then $F(T)=F(P(T))$.

Proof The inclusion $F(T) \subseteq F(P(T))$ is trivial. Let us show the reverse if $x \in K$ such that $P(T)(x)=\lambda_{0} x+\lambda_{1} T(x)+\cdots+\lambda_{n} T^{n}(x)=x$. Thus, $x \in \operatorname{co}\left\{T(x), \ldots, T^{n}(x)\right\}$. Now, assume that $\delta(O(x, n))>0$. Since $\lambda_{1}>0$, then $\lambda_{0} \neq 1$, consequently.

$$
x=\frac{\lambda_{1}}{\left(1-\lambda_{0}\right)} T(x)+\left(1-\frac{\lambda_{1}}{\left(1-\lambda_{0}\right)}\right) z
$$

where $z=\sum_{i=1}^{n-1} \sigma_{i} T^{i+1}(x)$ with $\sigma_{i}=\frac{\lambda_{i+1}}{1-\lambda_{0}-\lambda_{1}}$ for all $i=1, \ldots, n-1$. Moreover, it is easy to observe that $\sum_{i=1}^{n-1} \sigma_{i}=1$ (since $\sum_{i=0}^{n} \lambda_{i}=1$ ), this implies that $z \in \operatorname{co}\left\{T^{2}(x), T^{3}(x), \ldots, T^{n}(x)\right\}$; 
thus

$$
\begin{aligned}
\delta(O(x, n))= & \left\|x-T_{1}^{r_{1}^{x}(T)}(x)\right\|=\left\|\frac{\lambda_{1}}{\left(1-\lambda_{0}\right)} T(x)+\left(1-\frac{\lambda_{1}}{\left(1-\lambda_{0}\right)}\right) z-T_{1}^{r_{1}^{x}(T)}(x)\right\| \\
\leq & \frac{\lambda_{1}}{\left(1-\lambda_{0}\right)}\left\|T(x)-T_{1}^{r_{1}^{x}(T)}(x)\right\|+\left(1-\frac{\lambda_{1}}{\left(1-\lambda_{0}\right)}\right)\left\|z-T^{r_{1}^{x}(T)}(x)\right\| \\
= & \frac{\lambda_{1}}{\left(1-\lambda_{0}\right)}\left\|T(x)-T_{1}^{r_{1}^{x}(T)}(x)\right\| \\
& +\left(1-\frac{\lambda_{1}}{\left(1-\lambda_{0}\right)}\right)\left\|\sum_{i=1}^{n-1} \sigma_{i} T^{i+1}(x)-\left(\sum_{i=1}^{n-1} \sigma_{i}\right) T_{1}^{r_{1}^{x}(T)}(x)\right\| \\
\leq & \frac{\lambda_{1}}{\left(1-\lambda_{0}\right)}\left\|T(x)-T_{1}^{r_{1}^{x}(T)}(x)\right\| \\
& +\left(1-\frac{\lambda_{1}}{\left(1-\lambda_{0}\right)}\right) \sum_{i=1}^{n-1} \sigma_{i}\left\|T^{i+1}(x)-T_{1}^{r_{1}^{x}(T)}(x)\right\| \\
\leq & \frac{\lambda_{1}}{\left(1-\lambda_{0}\right)}\left\|T(x)-T_{1}^{r_{1}^{x}(T)}(x)\right\|+\left(1-\frac{\lambda_{1}}{\left(1-\lambda_{0}\right)}\right)\left(\sum_{i=1}^{n-1} \sigma_{i}\right) \delta(O(x, n)) .
\end{aligned}
$$

Since $\sum_{i=1}^{n-1} \sigma_{i}=1$, it follows that

$$
\frac{\lambda_{1}}{\left(1-\lambda_{0}\right)} \delta(O(x, n)) \leq \frac{\lambda_{1}}{\left(1-\lambda_{0}\right)}\left\|T(x)-T^{r_{1}^{x}(T)}(x)\right\|
$$

This gives that $\delta(O(x, n))=\left\|T(x)-T_{1}^{r_{1}^{x}(T)}(x)\right\|$ and, consequently, $\delta(O(T(x), n-1))=\| T(x)-$ $T^{r_{1}^{x}(T)}(x) \|$, which contradicts the fact that $r_{2}^{x}(T)>r_{1}^{x}(T)$. Thus $\delta(O(x, n))=0$, which gives that $T(x)=x$ and, consequently, $x \in F(T)$.

Remark 2.6 We note also that the condition $\lambda_{1}>0$ is crucial as the following example shows.

Example 2.7 Let $T:[0,1] \longrightarrow[0,1]$ be defined as follows: $T(x)=1-x$. It is easy to observe that $T$ is nonexpansive and $x_{0}=\frac{1}{2}$ is the unique fixed point of $T$. Now consider the polynomial $P(z)=\frac{1}{2} z^{2}+\frac{1}{2}$. We obtain that

$$
\begin{aligned}
& P(T):[0,1] \longrightarrow[0,1], \\
& x \longmapsto P(T)(x)=x .
\end{aligned}
$$

In this case, we observe that $F(P(T))=[0,1]$ and, consequently, we have $F(T) \subsetneq F(P(T))$.

By the same techniques as in the proof of Proposition 2.5, we prove the following proposition.

Proposition 2.8 If $K$ is a nonempty, bounded and convex subset in a normed space $X$ and $T \in \Xi_{K}$ with $k=1$ for all $n \in \mathbb{N}, n \geq 2$ and all $x \in K$, then $F(T)=F(P(T)$ ). 
Proof Indeed, in this case, the last inequality in the proof of Proposition 2.5 becomes

$$
\frac{\lambda_{1}}{\left(1-\lambda_{0}\right)} \delta(O(x, n)) \leq \frac{\lambda_{1}}{\left(1-\lambda_{0}\right)}\left\|T(x)-T^{r_{1}^{x}(T)}(x)\right\| \leq \frac{\lambda_{1}}{\left(1-\lambda_{0}\right)}\|x-T(x)\|,
$$

which gives that $\delta(O(x, n))=\|x-T(x)\|$ and shows that $r_{1}^{x}(T)=1$. By replacing in this inequality, we get also $\delta(O(x, n))=0$, which gives the desired result.

Now, we give the following important preparatory result.

Proposition 2.9 Let $K$ be a nonempty subset of a normed space $X$, and let $T$ be a selfmapping defined on $K$ satisfying that for all $(x, y) \in K \times K, x \neq y$, if $y=T(x)$, we have necessarily $x \neq T(y)$ together with the following condition:

$$
\|T(x)-T(y)\| \leq \frac{\Phi_{1}(\|x-T(x)\|,\|x-T(y)\|)+\Phi_{2}(\|y-T(y)\|,\|y-T(x)\|)}{\Phi_{3}(\|x-T(y)\|,\|y-T(x)\|)}
$$

for all $x, y \in K$, where:

(i) $\Phi_{1}, \Phi_{2}:[0,+\infty[\times[0,+\infty[\longrightarrow[0,+\infty[$ nondecreasing mappings relative to the first variable and $\Phi_{3}:[0,+\infty[\times[0,+\infty[\longrightarrow[0,+\infty[$ such that

$\Phi_{3}\left(t_{2}, t_{3}\right)=0 \Longleftrightarrow\left(t_{2}, t_{3}\right)=(0,0)$;

(ii) $\Phi_{2}(t, 0)=0$ for all $t \geq 0$;

(iii) $\frac{\Phi_{1}\left(t_{1}, t_{2}\right)+\Phi_{2}\left(t_{1}, t_{3}\right)}{\Phi_{3}\left(t_{2}, t_{3}\right)} \leq t_{1}$ for all $t_{1} \geq 0$ and $\left(t_{2}, t_{3}\right) \in[0,+\infty[\times[0,+\infty[\backslash\{(0,0)\}$.

Then

$$
\left\|T^{n}(x)-T^{n+1}(x)\right\| \leq\left\|T^{n-1}(x)-T^{n}(x)\right\|
$$

and for all strictly positive integers $m$ and $n$, we have

$$
\left\|T^{n}(x)-T^{m}(x)\right\| \leq\|x-T(x)\| .
$$

In particular $T \in \Xi_{K}$.

Proof Let $x \in K$, then

$$
\begin{aligned}
\left\|T^{n}(x)-T^{n+1}(x)\right\|= & \left\|T\left(T^{n-1}(x)\right)-T\left(T^{n}(x)\right)\right\| \\
\leq & \frac{\Phi_{1}\left(\left\|T^{n-1}(x)-T^{n}(x)\right\|,\left\|T^{n-1}(x)-T^{n+1}(x)\right\|\right)}{\Phi_{3}\left(\left\|T^{n-1}(x)-T^{n+1}(x)\right\|,\left\|T^{n}(x)-T^{n}(x)\right\|\right)} \\
& +\frac{\Phi_{2}\left(\left\|T^{n}(x)-T^{n+1}(x)\right\|,\left\|T^{n}(x)-T^{n}(x)\right\|\right)}{\Phi_{3}\left(\left\|T^{n-1}(x)-T^{n+1}(x)\right\|,\left\|T^{n}(x)-T^{n}(x)\right\|\right)} .
\end{aligned}
$$

Applying (ii) and (iii), we get

$$
\begin{aligned}
\left\|T^{n}(x)-T^{n+1}(x)\right\| & \leq\left\|T^{n-1}(x)-T^{n}(x)\right\| \\
& \vdots \\
& \leq\|x-T(x)\| .
\end{aligned}
$$


Now

$$
\begin{aligned}
\left\|T^{n}(x)-T^{m}(x)\right\|= & \left\|T\left(T^{n-1}(x)\right)-T\left(T^{m-1}(x)\right)\right\| \\
\leq & \frac{\Phi_{1}\left(\left\|T^{n-1}(x)-T^{n}(x)\right\|,\left\|T^{n-1}(x)-T^{m}(x)\right\|\right)}{\Phi_{3}\left(\left\|T^{n-1}(x)-T^{m}(x)\right\|,\left\|T^{m-1}(x)-T^{n}(x)\right\|\right)} \\
& +\frac{\Phi_{2}\left(\left\|T^{m-1}(x)-T^{m}(x)\right\|,\left\|T^{m-1}(x)-T^{n}(x)\right\|\right)}{\Phi_{3}\left(\left\|T^{n-1}(x)-T^{m}(x)\right\|,\left\|T^{m-1}(x)-T^{n}(x)\right\|\right)} .
\end{aligned}
$$

Using the first assertion together with the fact that $\Phi_{1}$ and $\Phi_{2}$ are nondecreasing relative to the first variable and (iii), we obtain that

$$
\begin{aligned}
\left\|T^{n}(x)-T^{m}(x)\right\| \leq & \frac{\Phi_{1}\left(\|x-T(x)\|,\left\|T^{n-1}(x)-T^{m}(x)\right\|\right)}{\Phi_{3}\left(\left\|T^{n-1}(x)-T^{m}(x)\right\|,\left\|T^{m-1}(x)-T^{n}(x)\right\|\right)} \\
& +\frac{\Phi_{2}\left(\|x-T(x)\|,\left\|T^{m-1}(x)-T^{n}(x)\right\|\right)}{\Phi_{3}\left(\left\|T^{n-1}(x)-T^{m}(x)\right\|,\left\|T^{m-1}(x)-T^{n}(x)\right\|\right)} \\
\leq & \|x-T(x)\| .
\end{aligned}
$$

This gives that $T$ satisfies (1) (here $k=1$ for all integers $n, m \geq 1$ and for all $x \in K$ ).

Definition 2.10 A bounded convex subset $K$ of a Banach space $X$ is said to have a normal structure if for each convex subset $M$ of $K$ with more than one point, there is a point $x \in M$ such that

$$
\sup \{\|x-y\|: y \in M\}<\delta(M)
$$

Under the assumptions of Proposition 2.9, in the following theorem we take $\Phi_{1}\left(t_{1}, t_{2}\right)=$ $\psi_{1}\left(t_{1}\right) \psi_{2}\left(t_{2}\right), \Phi_{2}\left(t_{1}, t_{2}\right)=\psi_{1}\left(t_{1}\right) \psi_{3}\left(t_{2}\right), \Phi_{3}\left(t_{1}, t_{2}\right)=\psi_{2}\left(t_{1}\right)+\psi_{3}\left(t_{2}\right)\left(t_{1}, t_{2} \geq 0\right)$, where $\psi_{1}, \psi_{2}$ and $\psi_{3}$ are self-mappings defined on $[0,+\infty[$.

Theorem 2.11 Let $K$ be a nonempty, bounded, closed, convex subset of a reflexive Banach space $X$ having a normal structure, and let $T$ be a continuous self-mapping defined on $K$ satisfying that for all $(x, y) \in K \times K, x \neq y$, if $y=T(x)$, we have necessarily $x \neq T(y)$ together with the following condition:

$$
\|T(x)-T(y)\| \leq \frac{\psi_{1}(\|x-T(x)\|) \psi_{2}(\|x-T(y)\|)+\psi_{1}(\|y-T(y)\|) \psi_{3}(\|y-T(x)\|)}{\psi_{2}(\|x-T(y)\|)+\psi_{3}(\|y-T(x)\|)}
$$

where $\psi_{1}$ is a strictly nondecreasing function and $\psi_{1}(t) \leq t$ for all $t \geq 0$.

Then $T$ has a unique fixed point in $K$.

Proof Since $X$ is a reflexive Banach space, then every descending family of nonempty closed convex subsets of $X$ has nonempty intersection. Then we can use the Zorn lemma to obtain a minimal subset $K_{1}$ of $K$ which is closed, convex and invariant under $T$.

- If $\delta\left(K_{1}\right)=0$, the problem is solved since in this case $K_{1}=\left\{x_{0}\right\}$, and thus $T\left(x_{0}\right)=x_{0}$.

- Assume that $\delta\left(K_{1}\right)>0$. 
Since $K$ has a normal structure, then there exists $y \in K_{1}$ such that

$$
\sup \left\{\|x-y\| ; x \in K_{1}\right\} \leq r<\delta\left(K_{1}\right)
$$

Hence, $\left\|y-T^{k}(y)\right\| \leq r$ for all $k \geq 1$ and, consequently, $\delta(O(y)) \leq r$. Let $H=\left\{x \in K_{1}\right.$ : $\delta(O(x)) \leq r\}$ and $G=\overline{\mathrm{co}}(T(H))$, which is a nonempty convex closed subset of $X$. Let $g \in G$. Then we will consider the following three cases.

First case: $g=T(h)$ for $h \in H$. Then

$$
\|g-T(g)\|=\left\|T(h)-T^{2}(h)\right\| \leq\|h-T(h)\| \leq r .
$$

Hence

$$
g \in H \quad \text { and } \quad T(g) \in G .
$$

Second case: $g=\sum_{i=1}^{n} \lambda_{i} T\left(h_{i}\right), h_{i} \in H, \lambda_{i} \geq 0, \sum_{i=1}^{n} \lambda_{i}=1$.

Then

$$
\begin{aligned}
\|g-T(g)\|= & \left\|T(g)-\sum_{i=1}^{n} \lambda_{i} T\left(h_{i}\right)\right\| \\
\leq & \sum_{i=1}^{n} \lambda_{i}\left\|T(g)-T\left(h_{i}\right)\right\| \\
\leq & \sum_{i=1}^{n} \lambda_{i} \frac{\psi_{1}(\|g-T(g)\|) \psi_{2}\left(\left\|g-T\left(h_{i}\right)\right\|\right)+\psi_{1}\left(\left\|h_{i}-T\left(h_{i}\right)\right\|\right) \psi_{3}\left(\left\|h_{i}-T(g)\right\|\right)}{\psi_{2}\left(\left\|g-T\left(h_{i}\right)\right\|\right)+\psi_{3}\left(\left\|h_{i}-T(g)\right\|\right)} \\
\leq & \sum_{i=1}^{n} \lambda_{i} \frac{\|g-T(g)\| \psi_{2}\left(\left\|g-T\left(h_{i}\right)\right\|\right)+r \psi_{3}\left(\left\|h_{i}-T(g)\right\|\right)}{\psi_{2}\left(\left\|g-T\left(h_{i}\right)\right\|\right)+\psi_{3}\left(\left\|h_{i}-T(g)\right\|\right)} \\
= & \|g-T(g)\|\left[\sum_{i=1}^{n} \lambda_{i} \frac{\psi_{2}\left(\left\|g-T\left(h_{i}\right)\right\|\right)}{\psi_{2}\left(\left\|g-T\left(h_{i}\right)\right\|\right)+\psi_{3}\left(\left\|h_{i}-T(g)\right\|\right)}\right] \\
& +r\left[\sum_{i=1}^{n} \lambda_{i} \frac{\psi_{3}\left(\left\|h_{i}-T(g)\right\|\right)}{\psi_{2}\left(\left\|g-T\left(h_{i}\right)\right\|\right)+\psi_{3}\left(\left\|h_{i}-T(g)\right\|\right)}\right] .
\end{aligned}
$$

We put

$$
\gamma_{1}=\left[\sum_{i=1}^{n} \lambda_{i} \frac{\psi_{2}\left(\left\|g-T\left(h_{i}\right)\right\|\right)}{\psi_{2}\left(\left\|g-T\left(h_{i}\right)\right\|\right)+\psi_{3}\left(\left\|h_{i}-T(g)\right\|\right)}\right]
$$

and

$$
\gamma_{2}=\left[\sum_{i=1}^{n} \lambda_{i} \frac{\psi_{3}\left(\left\|h_{i}-T(g)\right\|\right)}{\psi_{2}\left(\left\|g-T\left(h_{i}\right)\right\|\right)+\psi_{3}\left(\left\|h_{i}-T(g)\right\|\right)}\right] .
$$

We observe that $\gamma_{1}+\gamma_{2}=1$, thus

$$
\|g-T(g)\|\left(1-\gamma_{1}\right) \leq r \gamma_{2},
$$


which gives that

$$
\|g-T(g)\| \leq r
$$

and, consequently, $g \in H$ and $T(g) \in G$.

Third case: If $g$ is a limit of the form $\sum_{i=1}^{n} \lambda_{i} T\left(h_{i}\right), h_{i} \in H, \lambda_{i} \geq 0, \sum_{i=1}^{n} \lambda_{i}=1$, then

$$
\begin{aligned}
\|g-T(g)\| \leq & \left\|T(g)-T\left(\sum_{i=1}^{n} \lambda_{i} T\left(h_{i}\right)\right)\right\| \\
& +\left\|g-\sum_{i=1}^{n} \lambda_{i} T\left(h_{i}\right)\right\|+\left\|T\left(\sum_{i=1}^{n} \lambda_{i} T\left(h_{i}\right)\right)-\sum_{i=1}^{n} \lambda_{i} T\left(h_{i}\right)\right\| .
\end{aligned}
$$

By using the second case, we have that for all $n \geq 1$,

$$
\left\|T\left(\sum_{i=1}^{n} \lambda_{i} T\left(h_{i}\right)\right)-\sum_{i=1}^{n} \lambda_{i} T\left(h_{i}\right)\right\| \leq r .
$$

Let $\epsilon>0$, taking into account the continuity of $T$, we can choose $n$ sufficiently large such that

$$
\left\|g-\sum_{i=1}^{n} \lambda_{i} T\left(h_{i}\right)\right\|+\left\|T(g)-T\left(\sum_{i=1}^{n} \lambda_{i} T\left(h_{i}\right)\right)\right\|<\epsilon
$$

and, consequently, we infer that

$$
\|g-T(g)\| \leq r+\epsilon, \quad \forall \epsilon>0
$$

which implies that

$$
\|g-T(g)\| \leq r .
$$

Hence $g \in H$ and $T(g) \in G$. But $K_{1}$ is minimal, thus $K_{1}=G$, it follows that $\delta\left(K_{1}\right)=\delta(G)$.

Now, by taking into account the fact that if $A$ is a subset of a Banach space $X$, we have $\delta(A)=\delta(\overline{\mathrm{co}}(A))$, then

$$
\begin{aligned}
\delta\left(K_{1}\right)= & \delta(\overline{\operatorname{co}}(T(H))) \\
= & \delta((T(H))) \\
= & \sup \{\|T(x)-T(y)\|: x, y \in H\} \\
\leq & \sup \left\{\frac{\psi_{1}(\|x-T(x)\|) \psi_{2}(\|x-T(y)\|)+\psi_{1}(\|y-T(y)\|) \psi_{3}(\|y-T(x)\|)}{\psi_{2}(\|x-T(y)\|)+\psi_{3}(\|y-T(x)\|)}:\right. \\
& x, y \in H\} \\
\leq & \sup \left\{\frac{\psi_{1}(r) \psi_{2}(\|x-T(y)\|)+\psi_{1}(r) \psi_{3}(\|y-T(x)\|)}{\psi_{2}(\|x-T(y)\|)+\psi_{3}(\|y-T(x)\|)}: x, y \in H\right\} \\
\leq & \psi_{1}(r) \leq r<\delta\left(K_{1}\right),
\end{aligned}
$$


which is a contradiction, then $\delta\left(K_{1}\right)=0$, which gives that $K_{1}=\left\{x_{0}\right\}$ and shows that $T$ has a unique fixed point in $K_{1}$.

Remark 2.12 If $\psi_{1}(t)=\psi_{2}(t)=\psi_{3}(t)=t$, we find the main result of [14].

\section{Applications}

We start this section by giving the concept of $\varphi$-quasi-nonexpansive mappings.

Definition 3.1 Let $T$ be a self-mapping defined on a metric space $(X, d)$. We say that $T: X \longrightarrow X$ is a $\varphi$-quasi-nonexpansive mapping if $F(T) \neq \emptyset$, and there exists a function $\varphi:[0,+\infty[\longrightarrow[0,+\infty[$ such that

$$
d(T(x), z) \leq \varphi(d(x, z))
$$

for all $x \in X, z \in F(T)$.

It is easy to observe that every contraction mapping is $\varphi$-quasi-nonexpansive with $\varphi(t)=$ $\alpha t, 0 \leq \alpha<1$ and $t \in[0,+\infty[$, but the converse is false in general as the following example shows.

Example 3.2 Let $\varphi:[0,+\infty[\longrightarrow[0,+\infty[$ be a comparison function, and let $X=\mathbb{R}$, then the mapping $T: \mathbb{R} \rightarrow \mathbb{R}$ defined by

$$
T(x)= \begin{cases}\varphi(|x|) \sin \left(\frac{1}{x}\right) & \text { if } x \neq 0 \\ 0 & \text { if } x=0\end{cases}
$$

is $\varphi$-quasi-nonexpansive but not a contraction mapping. This implies that the class of $\varphi$ quasi-nonexpansive mappings contains strictly that of contraction mappings. In the case where $\varphi=I d_{X}$, we obtain the concept of quasi-nonexpansive mappings introduced by Tricomi [15] and studied by Diaz and Metcalf $[11,16]$ (for more details on the class of $\varphi$-quasinonexpansives, we can refer to $[17,18])$.

Definition 3.3 A function $\Phi:\left[0,+\infty\left[\longrightarrow\left[0,+\infty\left[\right.\right.\right.\right.$ is called subadditive if for all $t_{1}, t_{2} \in$ $\left[0,+\infty\left[\right.\right.$, we have $\Phi\left(t_{1}+t_{2}\right) \leq \Phi\left(t_{1}\right)+\Phi\left(t_{2}\right)$.

Theorem 3.4 Under assumptions of Theorem 2.11, if $\psi_{1}$ is a subadditive function with $\psi_{1} \leq \min \left\{\psi_{2}, \psi_{3}\right\}$, then $T$ is a $\psi_{2}$-quasi-nonexpansive self-mapping on $K$.

Proof Let $z$ be the unique fixed point of $T$ in $K$ and $x \in K$ with $x \neq z$, then

$$
\begin{aligned}
\|T(x)-z\| & =\|T(x)-T(z)\| \leq \frac{\psi_{1}(\|x-T(x)\|) \psi_{2}(\|x-z\|)+\psi_{1}(\|z-z\|) \psi_{3}(\|z-T(x)\|)}{\psi_{2}(\|x-z\|)+\psi_{3}(\|z-T(x)\|)} \\
& \leq \frac{\psi_{1}(\|x-T(x)\|) \psi_{2}(\|x-z\|)}{\psi_{2}(\|x-z\|)+\psi_{3}(\|z-T(x)\|)} \\
& \leq \frac{\psi_{1}(\|x-T(x)\|) \psi_{2}(\|x-z\|)}{\psi_{1}(\|x-T(x)\|)} \\
& =\psi_{2}(\|x-z\|) .
\end{aligned}
$$


Several iterative processes have been defined by many mathematicians. Some of them are the following.

Picard iteration: Let $(X,\|\cdot\|)$ be a normed space and $T: X \longrightarrow X$ be a self-mapping. Let $x_{0} \in X$ be fixed, we define the sequence $\left\{x_{n}\right\}_{n}$ recursively by

$$
x_{n+1}=T\left(x_{n}\right)=T^{n+1}\left(x_{0}\right) \quad \text { for all } n \in \mathbb{N} .
$$

Mann iteration ([19]): If $(X,\|\cdot\|)$ is a normed space, Mann iteration is defined by the following algorithm:

$$
x_{n+1}=\left(1-\alpha_{n}\right) x_{n}+\alpha_{n} T\left(x_{n}\right) \quad \text { for all } n \in \mathbb{N} \text {, }
$$

where $x_{0} \in X$ and $\left\{\alpha_{n}\right\}_{n} \subset[0,1]$.

Ishikawa iteration ([20]): Another iterative process of interest is the Ishikawa scheme of two steps given as follows: Let $x_{0} \in X$ be fixed, consider the sequence $\left\{x_{n}\right\}_{n}$ defined by

$$
\left\{\begin{array}{l}
y_{n}=\left(1-\beta_{n}\right) x_{n}+\beta_{n} T\left(x_{n}\right), \\
x_{n+1}=\left(1-\alpha_{n}\right) x_{n}+\alpha_{n} T\left(y_{n}\right) \quad \text { for all } n \in \mathbb{N},
\end{array}\right.
$$

where $\left\{\alpha_{n}\right\}_{n}$ and $\left\{\beta_{n}\right\}_{n}$ are sequences in $[0,1]$.

In the case where $\beta_{n}=0$, the Ishikawa iteration reduces to the Mann iteration. In general, there is no dependence between convergence results for Picard, Mann and Ishikawa iterations. However, some partial results on the equivalence of these processes have been given by Rhoades and Soltuz (see [21-26]).

Kirk iteration ([27]): Let $(X,\|\cdot\|)$ be a normed space, $K$ be a closed, convex, bounded subset of $X$. Let $T$ be a self-mapping defined on $K$. For each $x \in K$, the sequence $\left\{S^{n}(x)\right\}$ defined by $S: K \longrightarrow K$, where

$$
S=\lambda_{0} I+\lambda_{1} T+\cdots+\lambda_{n} T^{n}, \quad \lambda_{i} \geq 0, \lambda_{1}>0, \sum_{i=0}^{n} \lambda_{i}=1,
$$

is said to be Kirk's iterative process.

By using Theorem 3.4 together with ([28], Theorem 3.7), we obtain the following result for the convergence of the iterative schemes of Mann and Ishikawa.

Proposition 3.5 Let $(X,\|\cdot\|)$ be a Banach space. Let $\left\{\alpha_{n}\right\}_{n}$ and $\left\{\beta_{n}\right\}_{n}$ be two real sequences in $[0,1]$ such that $\left\{\alpha_{n} \beta_{n}\right\}_{n}$ converges to some positive real number, let $x_{0} \in X$. Under the assumptions of Theorem 3.4 with $\psi_{2}$ a comparison continuous function, the Ishikawa sequence given by (6) converges to the unique fixed point of T. Moreover, if $\left\{\beta_{n}\right\}_{n}$ is the constant sequence equal to 0 , the Mann iteration given by (5) converges to the same unique fixed point of $T$.

Definition 3.6 Let $A$ be a bounded subset of a normed space $(X,\|\cdot\|)$. The Kuratowski measure of noncompactness of $A$ denoted by $\alpha(A)$ is defined as the infimum of all $\epsilon>$ 0 such that $A$ admits a finite covering consisting of subsets with diameter less than $\epsilon$. A continuous mapping $T: X \longrightarrow X$ is said to be densifying if for every bounded subset $A$ of $X$ such that $\alpha(A)>0$, we have $\alpha(T(A))<\alpha(A)$. 
We denote by $\bar{B}_{X}$ the closed unit ball of the normed space $X$. The function $\alpha$ defined on the set of all subsets of $X$ denoted by $P(X)$ enjoys the following properties:

(a) regularity: $\alpha(\Omega)=0$ if and only if $\Omega$ is totally bounded;

(b) nonsingularity: $\alpha$ is equal to zero on every one-element set;

(c) monotonicity: $\Omega_{1} \subseteq \Omega_{2}$ implies $\alpha\left(\Omega_{1}\right) \leq \alpha\left(\Omega_{2}\right)$;

(d) semi-additivity: $\alpha\left(\Omega_{1} \cup \Omega_{2}\right)=\max \left\{\alpha\left(\Omega_{1}\right), \alpha\left(\Omega_{2}\right)\right\}$;

(e) Lipschitzianity: $\left|\alpha\left(\Omega_{1}\right)-\alpha\left(\Omega_{2}\right)\right| \leq 2 \rho\left(\Omega_{1}, \Omega_{2}\right)$, where $\rho$ denotes the Hausdorff semi-metric $\rho\left(\Omega_{1}, \Omega_{2}\right)=\inf \left\{\epsilon>0: \Omega_{1}+\epsilon \bar{B}_{X} \supset \Omega_{2}, \Omega_{2}+\epsilon \bar{B}_{X} \supset \Omega_{1}\right\}$;

(f) continuity: For any $\Omega \in P(X)$ and any $\epsilon$, there exists $\delta>0$ such that $\left|\alpha(\Omega)-\alpha\left(\Omega_{1}\right)\right|<\epsilon$ for all $\Omega_{1}$ satisfying $\rho\left(\Omega, \Omega_{1}\right)<\delta$;

(g) semi-homogeneity: $\alpha(t \Omega)=|t| \alpha(\Omega)$ for any number $t$;

(h) algebraic semi-additivity: $\alpha\left(\Omega_{1}+\Omega_{2}\right) \leq \alpha\left(\Omega_{1}\right)+\alpha\left(\Omega_{2}\right)$;

(i) invariance under translations: $\alpha\left(\Omega+x_{0}\right)=\alpha(\Omega)$ for any $x_{0} \in X$.

The following two theorems are omitted.

Theorem 3.7 ([29], Theorem 1.1.5) The Kuratowski measure of noncompactness is invariant under passage to the closure and to the convex hull: $\alpha(\Omega)=\alpha(\bar{\Omega})=\alpha(\operatorname{co}(\Omega))$.

Let us recall the following theorem due to Diaz and Metcalf [11].

Theorem 3.8 Let $T$ be a continuous self-mapping of the metric space $(X, d)$ such that

(i) $F(T)$ is nonempty;

(ii) for each $y \in X$ such that $y \notin F(T)$ and for each $z \in F(T)$, we have

$$
d(T(y), z)<d(y, z)
$$

Then one and only one of the following properties holds:

(a) for each $x_{0} \in X$, the Picard sequence $\left\{T^{n}\left(x_{0}\right)\right\}$ contains no convergent subsequences;

(b) for each $x_{0} \in X$, the sequence $\left\{T^{n}\left(x_{0}\right)\right\}$ converges to a point belonging to $F(T)$.

Theorem 3.9([30]) Let $T: K \longrightarrow K$ be a densifying mapping defined on a closed, bounded, convex subset $K$ of a Banach space $X$. Then $T$ has at least one fixed point.

Let us define the geometric property $P\left(f_{1}, f_{2}, f_{3}\right)$ given as follows.

Let $(X,\|\cdot\|)$ be a normed space, we say that $X$ has the property $P\left(f_{1}, f_{2}, f_{3}\right)$ if for all $x, y \in X$, $f_{1}(\|x+y\|)=f_{2}(\|x\|)+f_{3}(\|y\|)$ and $x \neq 0, y \neq 0$, then $x=c y(c>0)$.

In the case where $f_{1}=f_{2}=f_{3}=I_{\mathbb{R}^{+}}$, this property is satisfied by strictly convex Banach spaces.

Let $(X,\|\cdot\|)$ be a Banach space and $K$ be a closed, bounded subset of $X$. Let $T: K \longrightarrow K$ be a self-mapping on $K$. We define the set $\sum_{T}$ as follows:

$$
\sum_{T}=\{\alpha x+\beta T(x): \alpha, \beta \in[0,1], \alpha+\beta=1, x \in K \backslash F(T)\} .
$$

As in the case of Theorem 2.11, in the following result we take $\Phi_{1}\left(t_{1}, t_{2}\right)=\psi_{1}\left(t_{1}\right) \psi_{2}\left(t_{2}\right)$, $\Phi_{2}\left(t_{1}, t_{2}\right)=\psi_{1}\left(t_{1}\right) \psi_{3}\left(t_{2}\right)$ and $\Phi_{3}\left(t_{1}, t_{2}\right)=\psi_{2}\left(t_{1}\right)+\psi_{3}\left(t_{2}\right)$. 
Theorem 3.10 Let $T: K \longrightarrow K$ be a densifying mapping defined on a closed, bounded, convex subset of a Banach space $X$ having the property $P\left(\psi_{1}, \psi_{2}, \psi_{3}\right)$, where $\psi_{2}(t) \leq t$ for all $t \geq 0$ and $\psi_{1}$ is a nondecreasing, subadditive function with $\psi_{1} \leq \min \left\{\psi_{2}, \psi_{3}\right\}$. Assume that $T$ satisfies (2) and $F(T) \cap \sum_{T}=\emptyset$. Then, for each $x \in K$, the sequence $\left\{S^{n}(x)\right\}$ converges to a fixed point of $T$.

Proof Following Theorem 3.9, it follows that $F(T) \neq \emptyset$. Moreover, $S$ is a densifying mapping. Indeed, if $A$ is a bounded subset of $K$ such that $\alpha(A)>0$, then

$$
S(A) \subseteq \lambda_{0} A+\lambda_{1} T(A)+\cdots+\lambda_{n} T^{n}(A) .
$$

Hence, by monotonicity, algebraic semi-additivity and semi-homogeneity properties, we get

$$
\alpha(S(A)) \leq \lambda_{0} \alpha(A)+\lambda_{1} \alpha(T(A))+\cdots+\lambda_{n} \alpha\left(T^{n}(A)\right) .
$$

The fact that $T$ is densifying shows that

$$
\begin{aligned}
& \alpha(T(A))<\alpha(A), \\
& \vdots \\
& \alpha\left(T^{n}(A)\right) \leq \alpha\left(T^{n-1}(A)\right) \leq \cdots \leq \alpha(T(A))<\alpha(A) ;
\end{aligned}
$$

and therefore

$$
\alpha(S(A))<\left(\lambda_{0}+\cdots+\lambda_{n}\right) \alpha(A)=\alpha(A) .
$$

Also, by use of Theorem 3.9, $F(S) \neq \emptyset$. Moreover, by taking account of Propositions 2.8 and 2.5 , it follows that $F(T)=F(S) \neq \emptyset$.

For $x \in K$, let

$$
A=\bigcup_{n=0}^{+\infty} S^{n}\{x\} .
$$

We have $S(A)=\bigcup_{n=1}^{+\infty} S^{n}\{x\} \subset A$ and, since $A=\{x\} \cup S(A)$, this gives that

$$
\begin{aligned}
\alpha(A) & =\max \{\alpha(\{x\}), \alpha(S(A))\} \\
& =\max \{0, \alpha(S(A))\} \\
& =\alpha(S(A)) .
\end{aligned}
$$

Since $S$ is densifying, we establish that $\alpha(A)=0$, by the property of regularity, we establish that $A$ is totally bounded and, consequently, $\bar{A}$ is compact (since $X$ is a Banach space); therefore, the sequence $\left\{S^{n}(x)\right\}$ contains a convergent subsequence.

Now, if $y$ is a fixed point of $T$ and $x \in K \backslash F(T)$, then $T(y)=y$ and $T(x) \neq y$; consequently,

$$
\|T(x)-y\| \leq \frac{\psi_{1}(\|x-T(x)\|) \psi_{2}(\|x-y\|)}{\psi_{2}(\|x-y\|)+\psi_{3}(\|y-T(x)\|)} \quad\left(\text { since } \psi_{1}(0)=0\right) .
$$


Moreover, the fact that $\psi_{1}$ is nondecreasing subadditive and $\psi_{1} \leq \min \left\{\psi_{2}, \psi_{3}\right\}$ gives that

$$
\psi_{1}(\|x-T(x)\|) \leq \psi_{2}(\|x-y\|)+\psi_{3}(\|y-T(x)\|) .
$$

The above inequality is strict, indeed, if

$$
\psi_{1}(\|x-T(x)\|)=\psi_{2}(\|x-y\|)+\psi_{3}(\|y-T(x)\|)
$$

Using the property $P\left(\psi_{1}, \psi_{2}, \psi_{3}\right)$ satisfied by the Banach space $X$, we get

$$
x-y=c(y-T(x)) \quad(c>0)
$$

which implies that $x+c T(x)=(1+c) y$ and, consequently, $y=\frac{1}{1+c} x+\frac{c}{1+c} T(x) \in \Sigma_{T}$, which is a contradiction. This gives that

$$
\|T(x)-y\|<\psi_{2}(\|x-y\|) \leq\|x-y\|
$$

which implies that

$$
\left\|T^{k}(x)-y\right\|<\|x-y\| .
$$

It follows that

$$
\begin{aligned}
\|S(x)-y\| & =\left\|\sum_{k=0}^{n} \lambda_{k} T^{k}(x)-y\right\| \\
& =\left\|\sum_{k=0}^{n} \lambda_{k} T^{k}(x)-\sum_{k=0}^{n} \lambda_{k} y\right\| \\
& \leq \sum_{k=0}^{n} \lambda_{k}\left\|T^{k}(x)-y\right\| \\
& <\sum_{k=0}^{n} \lambda_{k}\|x-y\|=\|x-y\| .
\end{aligned}
$$

Then $S$ satisfies conditions of the theorem of Diaz and Metcalf, which implies that $\lim _{n \rightarrow+\infty} S^{n}(x)$ exists and belongs to $F(T)=F(S)$.

As an immediate consequence of the proof of Theorem 3.10, we have the following.

Theorem 3.11 Let $(X,\|\cdot\|)$ be a reflexive Banach space having the property $P\left(\psi_{1}, \psi_{2}, \psi_{3}\right)$, where $\psi_{2}(t) \leq t$ for all $t \geq 0$ and $\psi_{1}$ is a nondecreasing, subadditive function with $\psi_{1} \leq$ $\min \left\{\psi_{2}, \psi_{3}\right\}$. Let $T: K \longrightarrow K$ be a self-mapping defined on a closed, bounded, convex subset $K$ with normal structure in $X$. Assume that $T$ satisfies assumptions of Theorem 2.11. If $x_{0} \notin \sum_{T}$ (where $x_{0}$ is the unique fixed point of $T$ in $K$ ) and $S$ is a densifying mapping, then, for each $x \in K$, the sequence $\left\{S^{n}(x)\right\}$ converges to $x_{0}$. 
Recall that for the case of numerical stability we say that a fixed point iteration process is numerically stable if small perturbation in the initial data induces a small influence of the computed value of the fixed point. For the remainder of our study, we need the following two definitions about stability of general iterative processes.

Definition 3.12 Let $(X,\|\cdot\|)$ be a normed space, $T: X \longrightarrow X$ be a self-mapping on $X$. Let $\left\{x_{n}\right\}_{n} \subset X$ be the sequence generated by an iteration involving $T$ and defined by

$$
x_{n+1}=f\left(T, x_{n}\right) \quad \text { for all } n \in \mathbb{N},
$$

where $x_{0} \in X$ and $f$ is some function. Assume that $\left\{x_{n}\right\}_{n}$ converges to a fixed point $z_{0}$ of $T$. Let $\left\{y_{n}\right\}_{n} \subset X$, and we define

$$
\epsilon_{n}:=\left\|y_{n+1}-f\left(T, y_{n}\right)\right\| \quad \text { for all } n \in \mathbb{N} \text {. }
$$

Then

(i) the iteration process (7) is said to be $T$-stable if $\lim _{n \rightarrow \infty} \epsilon_{n}=0$ implies $\lim _{n \rightarrow \infty} y_{n}=z_{0}$

(ii) the iteration process (7) is said to be almost $T$-stable if $\sum_{n \in \mathbb{N}} \epsilon_{n}<\infty$ implies $\lim _{n \rightarrow \infty} y_{n}=z_{0}$.

For more information and interesting comments on these notions of stability, we can see [31]. On the other hand, it is easy to observe that an iterative process (7) which is $T$-stable is almost $T$-stable but the converse is not true in general (see the counter example given in [32]). Furthermore, the iterative processes can converge without being stable. Indeed, the following example given in $[32,33]$ confirms this.

Example 3.13 Let $(X,\|\cdot\|)=(\mathbb{R},|\cdot|)$ and $T:[0,1] \longrightarrow[0,1], T(x)=x$. It is easy to observe that $F(T)=[0,1]$.

The case of Picard iteration: Let $\left.\left.z_{0} \in\right] 0,1\right]$, and $x_{n+1}=T\left(x_{n}\right)=T^{n+1}\left(x_{0}\right)$ is a stationary sequence which is equal to $x_{0}$, this implies that its limit is $x_{0}$. On the other hand, if we take $y_{0}=0$ and $y_{n}=\frac{1}{n}$ for $n \geq 1$, we obtain that

$$
\left|y_{n+1}-T\left(y_{n}\right)\right|=\frac{1}{n(n+1)} \longrightarrow 0,
$$

but $\lim _{n \rightarrow+\infty} y_{n}=0 \neq z_{0}$. This shows that the Picard iteration converges but is not $T$ stable.

The case of Mann iteration: Let $\left.\left.z_{0} \in\right] 0,1\right],\left\{\alpha_{n}\right\}_{n} \subseteq[0,1]$ and $x_{n+1}=\left(1-\alpha_{n}\right) x_{n}+\alpha_{n} x_{n}=$ $x_{n}=x_{0}$ for all $n \geq 1$. Let $x_{0}=y_{0}$ and $y_{n}=\frac{1}{n+1}$. Thus

$$
\epsilon_{n}=\left|y_{n+1}-\left(1-\alpha_{n}\right) y_{n}-\alpha_{n} T\left(y_{n}\right)\right|=\frac{1}{(n+1)(n+2)} \longrightarrow 0,
$$

but $\lim _{n \rightarrow+\infty} y_{n}=0 \neq z_{0}$. This shows that the Mann iteration converges but is not $T$-stable.

In the following result, we establish almost stability of Picard's iterative process for our context of self-mappings. 
Corollary 3.14 Let $(X,\|\cdot\|)$ be a reflexive Banach space and $K$ be a nonempty, closed, convex and bounded subset of $X$ with normal structure. Suppose that the assumptions of Theorem 3.4 are satisfied with $\psi_{2}$ a continuous comparison function. If $z_{0}$ is the unique fixed point of $T$ and $x_{0} \in K$ with $x_{n+1}:=T\left(x_{n}\right), n \in \mathbb{N}$ is the Picard process and $\left\{y_{n}\right\}_{n} \subset K$. Define $\left\{\epsilon_{n}\right\}_{n}$ by

$$
\epsilon_{n}:=\left\|y_{n+1}-T\left(y_{n}\right)\right\| \quad \text { for all } n \in \mathbb{N} .
$$

If $\sum_{n \in \mathbb{N}} \epsilon_{n}<\infty$, then $\lim _{n \rightarrow \infty} y_{n}=z_{0}$. In other words, the Picard process is almost $T$-stable.

Proof The result is established by combining the fact that $T$ is $\psi_{2}$-quasi-nonexpansive together with Theorem 4.5 in [28].

\section{Competing interests}

The authors declare that they have no competing interests.

\section{Authors' contributions}

All authors contributed equally and significantly in writing this article. All authors read and approved the final manuscript.

\section{Acknowledgements}

The authors would like to thank the anonymous referees and the editor for their valuable comments which helped to improve this paper.

Received: 20 March 2015 Accepted: 28 August 2015 Published online: 24 September 2015

\section{References}

1. Abbas, M, Nazir, T, Aydi, H: Fixed points of generalized graphic contraction mappings in partial metric spaces endowed with a graph. J. Adv. Math. Stud. 6(2), 130-139 (2013)

2. Caristi, J: Fixed point theorems for mappings satisfying inwardness condition. Trans. Am. Math. Soc. 215, 241-251 (1976)

3. Karapınar, E: Fixed point theory for cyclic $\varphi$-contraction. Appl. Math. Lett. 24, 822-825 (2011)

4. Karapınar, E, Abdeljawad, T, Tas, K: A generalized contraction principle with control functions on partial metric spaces. Comput. Math. Appl. 3(73), 716-719 (2012)

5. Redjel, N: On some extensions of Banach's contraction principle and applications to the convergence and stability of some iterative processes. Adv. Fixed Point Theory 4, 555-570 (2014)

6. Rhoades, BE: A comparison of various definitions of contractive mappings. Trans. Am. Math. Soc. 226, $257-290$ (1977)

7. Sehgal, V: On fixed and periodic points for a class of mappings. J. Lond. Math. Soc. 5, 571-576 (1972)

8. Boyd, DW, Wong, JS: On nonlinear contractions. Proc. Am. Math. Soc. 20, 458-464 (1969)

9. Jaggi, DS: Some unique fixed point theorems. Indian J. Pure Appl. Math. 8, 223-230 (1977)

10. Dass, BK, Gupta, S: An extension of Banach contraction principle through rational expression. Indian J. Pure Appl. Math. 6, 1455-1458 (1975)

11. Diaz, JB, Metcalf, FT: On the set of subsequential limit points of successive approximations. Trans. Am. Math. Soc. 135, 459-485 (1969)

12. Kirk, WA: A fixed point theorem for mappings which do not increase distances. Am. Math. Mon. 76, 1004-1007 (1965)

13. Berinde, V: Iterative Approximation of Fixed Points. Springer, Berlin (2007)

14. Ray, BK, Singh, SP: Fixed point theorems in Banach space. Indian J. Pure Appl. Math. 9, $216-221$ (1978)

15. Tricomi, F: Un teorema sulla convergenza delle successioni formate delle successive iterate di una funzione di una variabile reale. G. Mat. Battaglini 54, 1-9 (1916)

16. Diaz, JB, Metcalf, FT: On the structure of the set of subsequential limit points of successive approximations. Bull. Am. Math. Soc. 73, 516-519 (1967)

17. Olatinwo, MO: Some stability results for nonexpansive and quasi-nonexpansive operators in uniformly convex Banach spaces using the Ishikawa iteration process. Carpath. J. Math. 24(1), 82-87 (2008)

18. Olatinwo, MO: Convergence and stability results for some iterative schemes. Acta Univ. Apulensis 26, 225-236 (2011)

19. Mann, WR: Mean value methods in iteration. Proc. Am. Math. Soc. 4, 506-510 (1953)

20. Ishikawa, S: Fixed point and iteration of a nonexpansive mapping in a Banach space. Proc. Am. Math. Soc. 59, 65-71 (1976)

21. Rhoades, BE, Soltuz, SM: On the equivalence of Mann and Ishikawa iteration methods. Int. J. Math. Math. Sci. 7, 451-459 (2003)

22. Rhoades, BE, Soltuz, SM: The equivalence of the Mann and Ishikawa iteration for non-Lipschitzian operators. Int. J. Math. Math. Sci. 42, 2645-2652 (2003)

23. Rhoades, BE, Soltuz, SM: The equivalence between the convergence of Ishikawa and Mann iterations for asymptotically pseudocontractive map. J. Math. Anal. Appl. 283, 681-688 (2003)

24. Rhoades, BE, Soltuz, SM: The equivalence of Mann and Ishikawa iterations for a Lipschitzian $\psi$-uniformly pseudocontractive and $\psi$-uniformly accretive maps. Tamkang J. Math. 35, 235-245 (2004) 
25. Rhoades, BE, Soltuz, SM: The equivalence between the convergence of Ishikawa and Mann iterations for asymptotically nonexpansive in the intermediate sense and strongly successively pseudocontractive maps. J. Math Anal. Appl. 289, 266-278 (2004)

26. Soltuz, SM: The equivalence of Picard, Mann and Ishikawa iterations with dealing with quasi-contractive operators. Math. Commun. 10, 81-89 (2005)

27. Kirk, WA: On successive approximations for nonexpansive mappings in Banach spaces. Glasg. Math. J. 12, 6-9 (1971)

28. Ruiz, DA: Convergence and stability of some iterative processes for a class of quasinonexpansive type mappings. J. Nonlinear Sci. Appl. 5, 93-103 (2012)

29. Akhmerov, RR, Kamenskii, MI, Potapov, AS, Rodkina, AE, Sadovskii, BN: Measures of Noncompactness and Condensing Operators. Birkhäuser, Basel (1992)

30. Furi, M, Vignoli, A: A fixed point theorem in complete metric spaces. Boll. Un. Mat. Ital. (4) 5, 505-509 (1969)

31. Olatinwo, MO: Some stability results for two hybrid fixed point iterative algorithms of Kirk-Ishikawa and Kirk-Mann type. J. Adv. Math. Stud. 1(1-2), 87-96 (2008)

32. Osilike, MO: Stability of the Mann and Ishikawa iteration procedures for $\phi$-strong pseudocontractions on nonlinear equations of the $\phi$-strongly accretive type. J. Math. Anal. Appl. 227, 319-334 (1998)

33. Hardy, G, Rogers, T: A generalization of a fixed point theorem of Reich. Can. Math. Bull. 16, 201-206 (1973)

\section{Submit your manuscript to a SpringerOpen ${ }^{\circ}$ journal and benefit from:}

- Convenient online submission

- Rigorous peer review

- Immediate publication on acceptance

- Open access: articles freely available online

- High visibility within the field

- Retaining the copyright to your article 